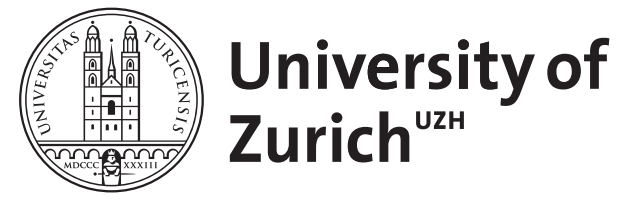

\title{
On convex hull violation by superpositions
}

Stoop, R

\begin{abstract}
Recently, we observed a violation of the convex hull principle for the super-imposed multifractals with measure, which we found difficult to explain in a simple way. Using the generalized entropy point of view for the description, we are able to resolve this problem
\end{abstract}

DOI: https://doi.org/10.1007/bf02732441

Posted at the Zurich Open Repository and Archive, University of Zurich ZORA URL: https://doi.org/10.5167/uzh-156331

Journal Article

Published Version

Originally published at:

Stoop, R (1997). On convex hull violation by superpositions. Journal of Statistical Physics, 88(5-6):13931398.

DOI: https://doi.org/10.1007/bf02732441 


\title{
On Convex Hull Violation by Superpositions
}

\author{
R. Stoop ${ }^{1}$
}

Received December 31, 1996

\begin{abstract}
Recently, we observed a violation of the convex hull principle for the superimposed multifractals with measure, which we found difficult to explain in a simple way. Using the generalized entropy point of view for the description, we are able to resolve this problem.
\end{abstract}

KEY WORDS: Fractals; phase transitions; entropy.

The theory of fractals ${ }^{(1-5)}$ has attracted much interest since singular measures of multifractal structure ${ }^{(6)}$ were shown to characterize strange attractors, ${ }^{(7)}$ diffusion ${ }^{(8,9)}$ and scattering processes, ${ }^{(4,10)}$ turbulence, fractal diffusion-limited aggregation, ${ }^{(3)}$ etc. The range of applications was recently extended to cover even more complex cases by considering so-called spatially extended systems. ${ }^{(11)}$ In a generalized sense, the system investigated below belongs to this class.

Often in experiments the full multifractal structure of the object cannot be accessed. What can be observed is a fractal structure which typically has a measure on it which is not proportional to its length scales. This measure can be the product of a partial overlap due to a projection from higher dimensional spaces. Or, the measure can arise quite naturally as the sum of different probability measures attached to the same Cantor structure. For this whole class of problems, only few theoretical results have been obtained.

In ref. 12, the superposition of two binary multifractals was considered and the behavior of the $f(\alpha)$-function ${ }^{(3,5-7)}$ was discussed for this system. It was shown that (in addition to first order phase transitions which can be

\footnotetext{
'Institut für Neuroinformatik, CH-8006 Zürich, Switzerland, and Institut für Theoretische Physik, Universität Zürich, CH-8057, Zürich-Irchel, Switzerland.
} 
expected to appear), transitions of second order emerge, under specific conditions which could not be formulated in "natural" terms. Along with the second order phase transition, a violation of the convex hull principle for entropy functions was observed. In the present contribution, this phenomenon is explained in a simple way using the generalized entropy point of view. The present discussion applies to a wider context because the generalized entropy point of view comprises all kinds of specific entropy properties and thus goes beyond the discussion in ref. 12. Suppose as in ref. 12 that the sources of the superposition are complete, self-similar $\tilde{M}$-scale multifractals. Each of these sources is then determined by the probabilities $p_{i}, i \in\{1,2, \ldots, \tilde{M}\}$, and the associated length scales $l_{i}{ }^{(1-3)}$ The hierarchic structure of this multiplicative process is captured in the generalized partition sum $^{(6,7,12-17)}$

$$
Z(q, \beta, N)=\sum_{j} p_{j}^{q} l_{j}^{\beta}
$$

where $N$ denotes the level of the construction hierarchy. Here $j \in \tilde{M}^{N}$ is the symbolic address of an allowed contribution to the hierarchic process (for multiplicative measures we have $p_{j}=p_{1} p_{1} p_{2} \ldots$ if $j=\{1,1,2, \ldots\}$ and allowed means that $p_{j} \neq 0$ ). The probabilities are normalized: $\sum_{i} p_{i}=1$. In order to label $M$ independent sources we use the index $k$. Accordingly, the system is characterized by the set of quantities $p_{i}(k), l_{i}(k), i=1, \ldots, \bar{M}$ and $k=1, \ldots, M$. In the following we consider the case of the superposition $\mu$ of $M$ multifractal measures, i.e., $\mu=\sum_{k} \mu(k)$, where $k=1, \ldots, M$. A simple situation arises if the supports of the $k$ measures are the same and the measures are multiplicative. This implies that $l_{i}(k)=l_{i}$ for all $i=1, \ldots, \tilde{M}$ and $k=1, \ldots, M$. For full binary (i.e., $\tilde{M}=2$ ) grammar the partition sum of the superposition has the form

$$
Z(q, \beta, N)=\sum_{j=0}^{N}\left(\begin{array}{c}
N \\
j
\end{array}\right)\left(\sum_{k=1}^{M} \pi(k) p_{1}(k)^{j} p_{2}(k)^{(N-j)}\right)^{q}\left(l_{1}^{j} l_{2}^{(N-j)}\right)^{\beta}
$$

In this formula the parameters $\pi(k), k=1,2$, are the weights of the contributions of the involved multifractals $\left(\sum_{k} \pi(k)=1\right)$. For $\tilde{M}>2$, the binomial coefficient has to be replaced by more involved expressions; nonfull grammars may sometimes be reduced to full grammars of different type. ${ }^{(17)}$ Letting $\xi=j / N$, in order to evaluate the partition sum, $Z$ is written as an integral ${ }^{(12)}$

$$
Z(q, \beta, N) \sim \int_{0}^{1} e^{-N g(\xi, q, \beta)} d \xi
$$


with, specifying for the simplest case $M=2, \tilde{M}=2$,

$$
\begin{aligned}
g(\xi, q, \beta)= & \xi \ln (\xi)+(1-\xi) \ln (1-\xi) \\
& +\left(\xi\left[-q \ln \left(p_{1}(1)\right)-\beta \ln \left(l_{1}\right)\right]\right. \\
& \left.+(1-\xi)\left[-q \ln \left(p_{2}(1)\right)-\beta \ln \left(l_{2}\right)\right]\right) \theta\left(\xi-\xi_{0}\right) \\
& +\left(\xi\left[-q \ln \left(p_{1}(2)\right)-\beta \ln \left(l_{1}\right)\right]\right. \\
& \left.+(1-\xi)\left[-q \ln \left(p_{2}(2)\right)-\beta \ln \left(l_{2}\right)\right]\right) \theta\left(\xi_{0}-\xi\right)
\end{aligned}
$$

where $\theta(x)$ denotes the step-function. The special value $\xi_{0}$ is characterized by the equality of the two contributions; it has the value $\xi_{0}=\left[1+\ln \left(p_{1}(1) /\right.\right.$ $\left.\left.p_{1}(2)\right) / \ln \left(p_{2}(1) / p_{2}(2)\right)\right]^{(-1)}$. Fractals reveal a deep connection to more traditional fields of physics due to a (formal) equivalence of multifractals with spin systems. ${ }^{(18)}$ In analogy to the latter, thermodynamic quantities are defined, and nonanalytic behavior of these functions can be interpreted as phase transition phenomena. ${ }^{(19)}$ Using this language, $F(q, \beta)=\lim _{N \rightarrow \infty}$ $\ln [Z(q, \beta, N)] / N$ is the generalized free energy, and we have $F(q, \beta)=$ $-g(\xi(q, \beta), q, \beta)$ where $\xi(q, \beta)$ determines the maximum of the integrand in Eq. (3) by minimizing the function $g$, for given $q$ and $\beta$. The generalized entropy function $S(\alpha, \varepsilon)^{(13)}$ arises as the Legendre transform of $F(q, \beta)$ as

$$
S(\alpha, \varepsilon)=F(q, \beta)+q \alpha \varepsilon+\beta \varepsilon
$$

Here, $\varepsilon=-\partial F(q, \beta) / \partial \beta$ is the local scaling rate of the support and the "local" dimension appears as $\alpha=-(\partial F(q, \beta) / \partial q) / \varepsilon$. Note that it may be preferable to change the sign of $\varepsilon$ in order to express a different interpretation of the length scales.

For the discussion of the generalized entropy spectrum we first recall that the general case without superposition does not show phase transitions. For a system of ternary grammar, a typical entropy function appears as a smooth sheet over the scaling plane $(\varepsilon, \alpha) \cdot{ }^{(13)}$ On a line in this sheet, the function $f(\alpha)$ is evaluated. It is a simple consequence of the definition of $f(\alpha):=S(\alpha, \varepsilon) /\left.\varepsilon\right|_{\beta(q): F(q, \beta(q))=0}$ that this line can be thought to emerge from a simple experiment: Hold a ruler parallel to the $\varepsilon$ axis, while the left end of the ruler is at the origin of the coordinate system. Move the ruler now towards higher $\alpha$-values, with one end still tied to the $(\varepsilon=0, \alpha)$-axis, without making any rotation with respect to the $\varepsilon$-axis. The tangential points of the ruler with the entropy surface form the line along which $f(\alpha)$ is evaluated. By a projection of this line the trace of $f(\alpha)$ in the $(\varepsilon, \alpha)$-plane is obtained. The entropy function of a single binary system degenerates into a sheet with one-dimensional support due to the fact that the function 
$(\beta, q) \rightarrow(\varepsilon, \alpha)$ is not injective ${ }^{(7,15)}$ (in the presence of phase transitions, a two-dimensional support can be obtained.)

Let us consider the $f(\alpha)$-curve of the superimposed system. In a naive approach, one would associate the convex hull of the individual entropy functions with the superimposed system. This, however, is not correct in the present case. If we consider the superposition of two binary systems, we start off with two sheets in the scaling plane $(\varepsilon, \alpha)$. These sheets have to intersect, and that there are only four cases: the two cases shown in Fig. 1 and two more cases obtained from their reflection about a line parallel to the $\alpha$-axis through their point of intersection. We will discuss only the situations shown in Fig. 1, for the two remaining cases analogous arguments apply. Each symbolic address $j$ triggers one specific logarithmic length scale $\varepsilon$. However, each of the length scales is associated with two different measure exponents $\alpha_{k}, k=1, \ldots, M=2$. The measure exponents correspond to probabilities, which themselves correspond to the measure on the Cantor piece labeled by $j$. In the asymptotic limit $N \rightarrow \infty$ the largest probability dominates (in our setting, $\sum p(j)_{k}^{N}$ and not $\left(\sum p(j)_{k}\right)^{N}$ is relevant
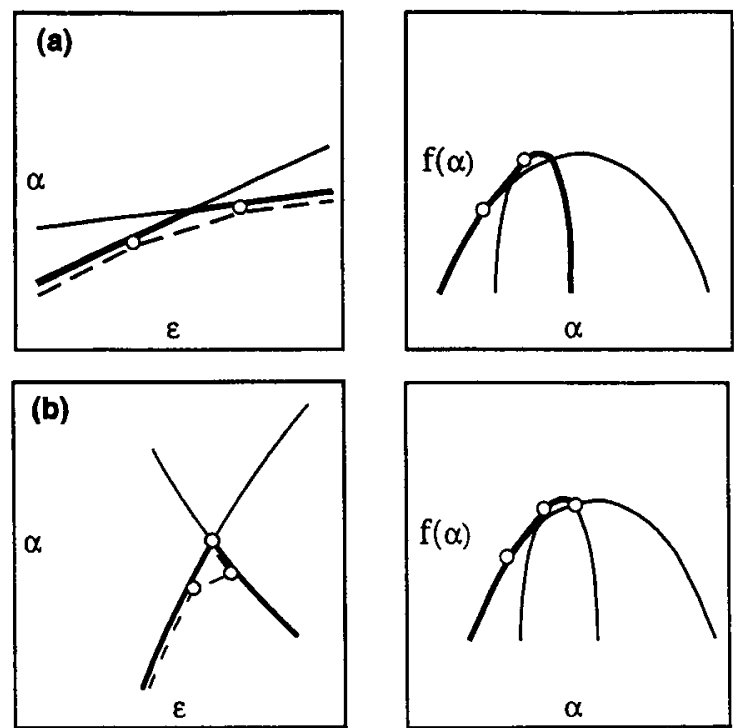

Fig. 1. Support obtained for a system of 2 binary sources (light lines) with relevant branches (heavy lines) in the $(\varepsilon, \alpha)$-plane. The $f(\alpha)$-spectrum is evaluated along the dashed lines; dots indicate the phase transition points. The graph of $f(\alpha)$ itself is shown by heavy lines; light lines indicate the $f(\alpha)$-curves associated with the two systems before superposition. (a) Both individual entropy sheets are ascending, (b) one is ascending, the other is descending.

Note: The $f(\alpha)$-curve of the superimposed system is not obtained from the convex hull of the contributions. 
for the measure!). The largest $p(j)_{k}$ corresponds to the smallest $\alpha(j)_{k}$. Therefore, only the smallest $\alpha$ will survive. As a consequence, the upper wings are cut off for the entropy function of the superimposed system.

If for the superimposed system $q$ is monitored from $\infty$ to $-\infty$, in Fig. 1 the ruler starts moving up the branch which provides the lowest $\alpha$-value. At $(q=1, \beta=0)$, invariably a phase transition appears, because at this parameter set the free energies of both branches are zero. On the two branches, the free energies grow at different rates, which yields a first order transition. ${ }^{(12)}$ The intersection point of the two branches is characterized by the equality of the free energies, the length- and the measure exponents, respectively. This point therefore corresponds to the second intersection point of the $f(\alpha)$-curves. In case (a) of Fig. 1, where both individual entropy curves are ascending, for $q \rightarrow-\infty$. $f \alpha$ follows the more narrow individual curve until zero entropy is reached. In case (b), the $f(\alpha)$-curve has to stop at the intersection point. Because of the above characterization of the intersection point, the point itself can be understood as a phase of its own in the free energy picture. Entering into this phase can therefore be interpreted as a second order phase transition. In both cases, the resulting entropy function is not obtained from the convex hull of the contributing individual $f(\alpha)$-functions.

In addition to $f(\alpha)$ other specific entropy functions can be considered, ${ }^{(16)}$ which all arise by restriction of $S(\varepsilon, \alpha)$ according to some conditions (e.g., the restriction to $\beta=0$ yields the Legendre transform $g(A)$ of the Rényi entropies $K(q)){ }^{(20)}$ Most of these specific entropy functions also undergo a first order transition. Actually, the whole area between the two branches is an area of first order transitions which has to be added to the traces shown in Fig. 1. We see this more clearly if we consider the superposition of $M=2$ ternary systems (see ref. 21). We note that for such a system, instead of the second order phase transition point, a transition line emerges. Increasing the number of contributing ternary maps with randomly chosen sets of probabilities yields an asymptotic entropy function. The convergence towards this asymptotic function is very fast (the form is established already for $M \sim 25$ ). When such "universal" entropy functions of different grammatical types are compared, it is found that universal entropy functions of simpler grammatical type are identical with a part of the universal entropy function of higher grammatical type. ${ }^{(22)}$

In conclusion we were able to explain a violation of the convex hull principle in a straightforward way. We found that the generalized entropy representation provides an excellent tool for the understanding of phase transition effects in multifractals. Our approach can be extended to cover other specific entropy functions; it is not restricted to the discussion of $f(\alpha)$. 


\section{ACKNOWLEDGMENT}

Stimulating discussions with G. Radons are gratefully acknowledged.

\section{REFERENCES}

1. B. B. Mandelbrot, The Fractal Geometry of Nature (W. H. Freeman, New York, 1982).

2. K. Falconer Fractal Geometry (J. Wiley and Sons, Chichester, 1990).

3. J. Feder, Fractals (Plenum, New York, 1988).

4. T. Tél, Transient Chaos (World Scientific, Singapore, 1991).

5. H. G. Schuster, Deterministic Chaos, 2nd ed. (VCH, Weinheim, 1989).

6. T. C. Halsey, M. H. Jensen, L. P. Kadanoff, I. Procaccia, and B. Shraiman, Phys. Rev. A 33:1141 (1986).

7. J. Peinke, J. Parisi, O. E. Roessler, and R. Stoop, Encounter with Chaos (Springer, Berlin, 1992).

8. R. Artuso, G. Casati, and D. L. Shepelyanski, Phys. Rev. Lett. 68:3826 (1992); R. Ketzmerick, G. Petschel, and T. Geisel, Phys. Rev. Lett. 69:695 (1992); I. Guarneri and G. Mantica, Phys. Rev. Lett. 73:3379 (1994).

9. R. Artuso, Phys. Lett. A 160:530 (1991); X.-J. Wang and C.-K. Hu, Phys. Rev. E 48:728 (1993); R. Stoop, Europhys. Lett. 25:99 (1994); R. Stoop, Europhys. Lett. 29:433 (1995).

10. C. Godrèche and I. M. Luck, J. Phys. A.: Math. Gen. 23:3769 (1990); F. Axel and H. Terauchi, Phys. Rev. Lett. 66:2223(1991).

11. L. A. Bunimovich and Ya. G. Sinai, Nonlinearity 1:491 (1988).

12. G. Radons and R. Stoop, J. Stat. Phys. 82:1063 (1996).

13. M. Kohmoto, Phys. Rev. A 37:1345(1988); J. Peinke, J. Parisi, O. E. Roessler, and R. Stoop, Encounter with Chaos (Springer, Berlin, 1992);

14. T. Tél, Z. Naturforsch. 43a:1154 (1988); Z. Kovács and T. Tél, Phys. Rev A 45:2270 (1992).

15. R. Stoop, Phys. Rev. A 46:7450 (1992).

16. R. Stoop, Phys. Rev. E 47:3927 (1993).

17. R. Stoop and J. Parisi, Physica D 58:325 (1992). For an approach starting from basics see R. Artuso, E. Aurell, and P. Cvitanović, Nonlinearity 3:361 (1990).

18. See e.g.: C. J. Thompson, Classical Equilibrium Statistical Mechanics (Clarendon Press, Oxford 1988).

19. D. Katzen and I. Procaccia, Phys. Rev. Lett. 58:1169 (1987), and refs therein; E. Ott, C. Grebogi, and J. A. Yorke, Phys. Lett. A 135:343 (1989).

20. A. Csordás, G. Gyórgyi, P. Széphalusy, and T. Tél, Chaos 3:31 (1993).

21. R. Stoop, in Nonlinear Physics of Complex Systems-Current Status and Future Trends, eds. J. Parisi, S. C. Müller, and W. Zimmermann (Springer, Heidelberg, 1996).

22. R. Stoop and W.-H. Steeb, Europhys. Lett. 35:177 (1996). 